\title{
Magnetic, Structural and Optical Properties of Nickel Doped Zinc Sulphide Nanoparticles
}

\author{
A. Maria Bernadette Leena ${ }^{1}$, K.Raji ${ }^{2}$ \\ ${ }^{1,2}$ Dept. of Physics, Holy Cross College (Autonomous), Tiruchirappalli. TamilNadu,India \\ Corresponding Author: leenavijay.jj@gmail.com.
}

Available online at: www.isroset.org

Received: 18/Apr/2019, Accepted: 12/May/2019, Online: 30/Jun/2019

\begin{abstract}
The wide bandgap metal sulfide semiconductors, such as $\mathrm{ZnS}$ when doped with transition metal ion $\mathrm{Ni}^{2+}$ have shown promises in versatile applications such as spintronics devices, solar energy conversion, optoelectronics, and photonics. Nickel doping leads to substantial modification in technology for ZnS semiconductors, as it suitably tunes the optical, electrical and magnetic properties of materials. Here $2 \%, 4 \%$, and $6 \%$ of Nickel ion $\left(\mathrm{Ni}^{2+}\right)$ is doped with $\mathrm{ZnS}$ nanoparticles, which were synthesized via a simple chemical precipitation route. The resultant nanoparticles were characterized by FTIR, UV-Vis spectroscopy, XRD, Photo luminance, SEM, and VSM. The results showed that all the nickel-doped ZnS samples were successfully obtained with a cubic structure with crystallites in $4.5 \mathrm{~nm}$ to $5.5 \mathrm{~nm}$ range. The band gap energies of the Nickel doped $\mathrm{ZnS}$ were also estimated by UV -visible absorbance spectra which is found to decrease with the increase of crystallite size. The SEM results showed the appearance cubic structure of the samples. The VSM study showed that these particles have room temperature ferromagnetism or superparamagnetism, essential for the discovery of new spintronic devices.
\end{abstract}

Keywords: Nickel doped ZnS Chalcogenides, co-precipitation, optical properties and superparamagnetic

\section{INTRODUCTION}

Discovery of a new class of spintronics devices based ferromagnetism has stimulated intensive research in the synthesis of diluted magnetic semiconductors (DMS) [1].The II-VI semiconducting chalcogenide nanoparticles, especially sulphides and selenides, called diluted magnetic semiconductors (DMS), having magnetic properties, have attracted the curiosity of researchers, because of the exchange interactions between the spins of the dopant atoms and the carriers in the semiconductor host, as they are expected to bring global ferromagnetic order in the entire lattice at room temperature [2]. Cubic $\mathrm{ZnS}$ has been recognized as an outstanding host semiconductor for sustaining room-temperature ferromagnetism with the direct band gap of $3.54 \mathrm{eV}[3,4]$. However, the doping ability of $\mathrm{ZnS}$ with transition metals like Nickel, Cobalt, Manganese, Iron has received considerable attention due to its optical and magnetic properties. $\mathrm{ZnS}$ based diluted magnetic semiconductors are good candidates for applications such as magneto-optical devices, magnetic field sensors, spintronics, magnetic recording and magneto-optical switches, solar cells and gas sensors.

In the present investigation, zinc sulfide nanoparticles with three different Nickel concentration $(2 \%, 4 \%, 6 \%)$ were synthesized by a simple chemical co-precipitation method.
Notable changes in the optical and magnetic properties of $\mathrm{ZnS}$ due to Nickel doping have been observed.

\section{MATERIAL AND METHODS}

\subsection{Synthesis and characterization}

Nickel doped $\mathrm{ZnS}$ nanoparticles were prepared by the aqueous co-precipitation method. The $0.1 \mathrm{M}$ aqueous solution of zinc acetate and nickel acetate were prepared with deionized water. $0.1 \mathrm{M}$ sodium sulfide $\left(\mathrm{Na}_{2} \mathrm{~S}\right)$ was also prepared deionized water with the precursor ratio of 1:1. The aqueous solution of $\mathrm{Na}_{2} \mathrm{~S}$ was added dropwise into the mixed solution of zinc acetate and nickel acetate at room temperature. A green-yellow colored solution is obtained. The solution was stirred for more than one hour to obtain a homogenous solution and allowed to precipitate. It was further washed several times with distilled water. The wet precipitate was dried and thoroughly ground using mortar to obtain the samples in the form of fine powder. Finally, the green colored $\mathrm{Ni}^{2+}$ doped $\mathrm{ZnS}$ nanoparticles were obtained.

The morphology of the powdered samples was studied by the scanning electron microscopy (SEM) analysis. The Fourier transform infrared studies of the samples were studied by SHIMADZU FTIR 8400S model spectrometer through the $\mathrm{KBr}$ method. The UV spectrum was taken in the absorbance mode in the wavelength range from $200 \mathrm{~nm}$ to 
$800 \mathrm{~nm}$. XRD line broadening method of particle size determination was chosen in this investigation for determining the crystallite size of the powder sample. The magnetic nature of the nickel-doped $\mathrm{ZnS}$ is investigated by vibrating magnetometer.

\section{III.RESULTS}

\subsection{XRD studies}

The XRD patterns of the $2 \%, 4 \%$, and $6 \%$ atomic percentage of the prepared samples of nickel-doped zinc sulfide nanoparticles are shown in figure 1. XRD studies reveal that the samples are nano-sized. This quantum size nature of the samples was revealed in the X-ray line broadening. The X-ray peaks have been found to correspond to (111), (220), and (311) planes of the pure $\mathrm{ZnS}$ cubic phase (JCPDS 05-0566). The structure of $\mathrm{ZnS}$ remains virtually unchanged by the incorporation of the nickel dopants but the peaks got broadened. In all patterns, no peaks corresponding to impurities were detected.

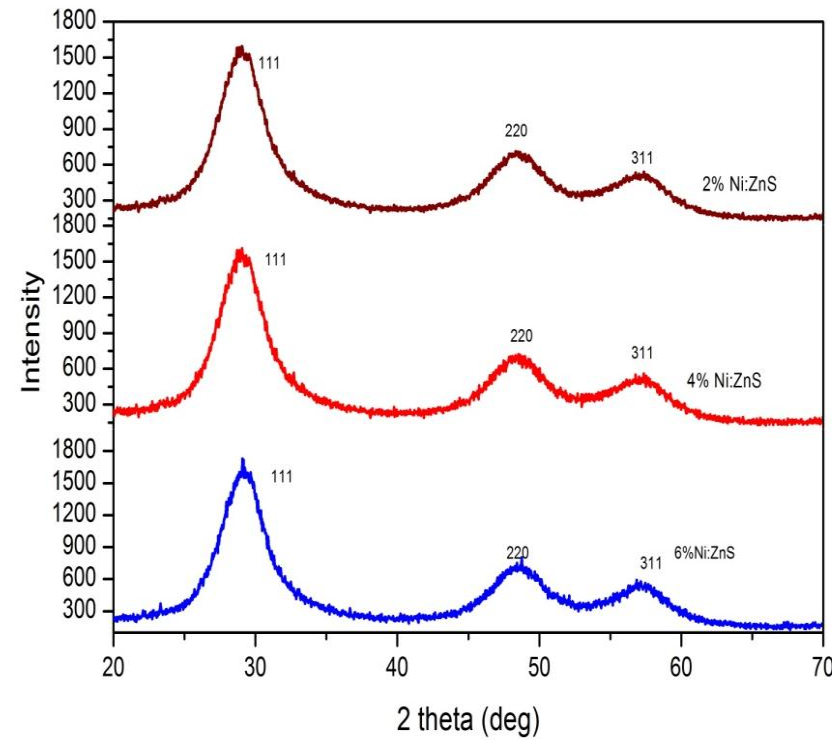

Figure 1: XRD patterns of nickel-doped $\mathrm{ZnS}$ at the different doping concentration

The size of the synthesized samples was calculated using the Debye Scherrer equation (1)

$$
D=\frac{0.9 \lambda}{\beta \operatorname{Cos} \theta}
$$

Where $\lambda$ represents the wavelength of Xrays, $\beta$ represents half a width of the full maximum and $\theta$ is the diffraction angle. The average grain size of the particle is found to be in 4 to $6 \mathrm{~nm}$.

The mean calculated crystallite size of the $\mathrm{ZnS}$ nanoparticles, with different atomic percentage Nickel shows that the synthesized nanoparticles are in the quantum confinement regime as given in table (1).
Table. 1 crystallite size and the bandgap of the nickeldoped $\mathrm{ZnS}$ nanoparticles

\begin{tabular}{|l|c|l|l|}
\hline $\begin{array}{l}\text { Sample } \\
\text { name }\end{array}$ & $\begin{array}{l}\text { The atomic } \\
\text { percentage of } \\
\text { doping agent }\end{array}$ & $\begin{array}{l}\text { Crystallitee } \\
\text { size from } \\
\text { eqn. }(1) \\
(\mathrm{nm})\end{array}$ & $\begin{array}{l}\text { Optical } \\
\text { band gap } \\
\left(\mathrm{E}_{\mathrm{g}}\right) \text { from } \\
\text { eqn. }(2) \\
\mathrm{eV}\end{array}$ \\
\hline $\begin{array}{l}\text { Ni: ZnS } \\
\text { sample 1 }\end{array}$ & $2 \%$ & 4.5 & 3.51 \\
\hline $\begin{array}{l}\text { Ni: ZnS } \\
\text { sample 2 }\end{array}$ & $4 \%$ & 5 & 3.49 \\
\hline $\begin{array}{l}\text { Ni: ZnS } \\
\text { sample 3 }\end{array}$ & $6 \%$ & 5.5 & 2.76 \\
\hline
\end{tabular}

\subsection{SEM studies}

Scanning electron microscopy was used to analyze the morphology and size of the synthesized samples
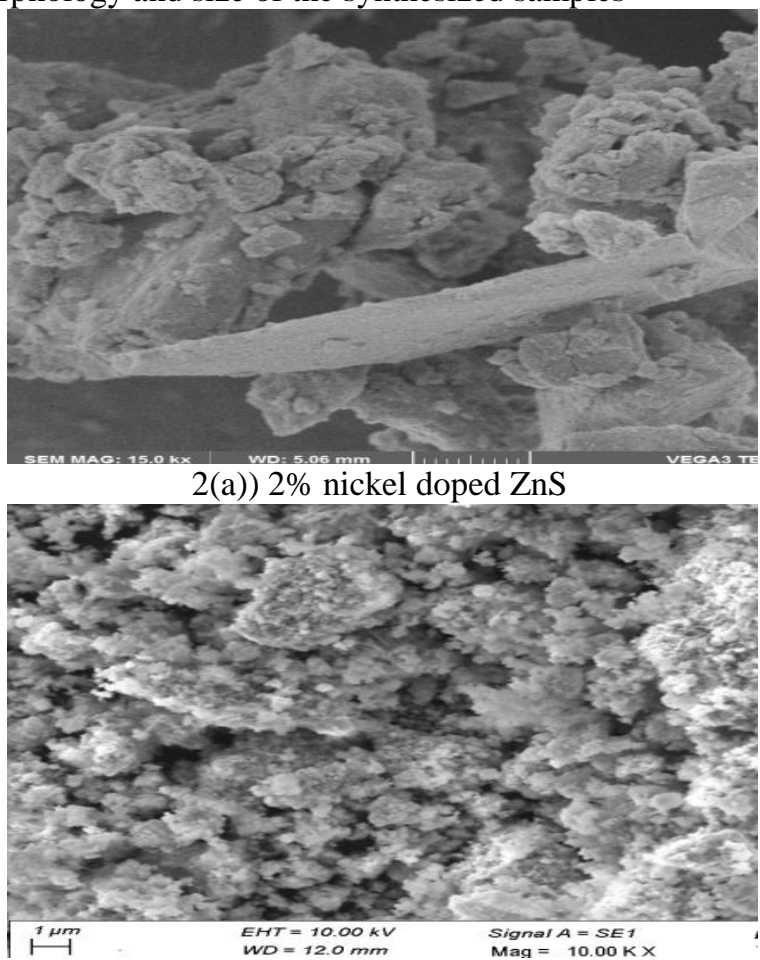

2(b) $4 \%$ Ni-doped $\mathrm{ZnS}$

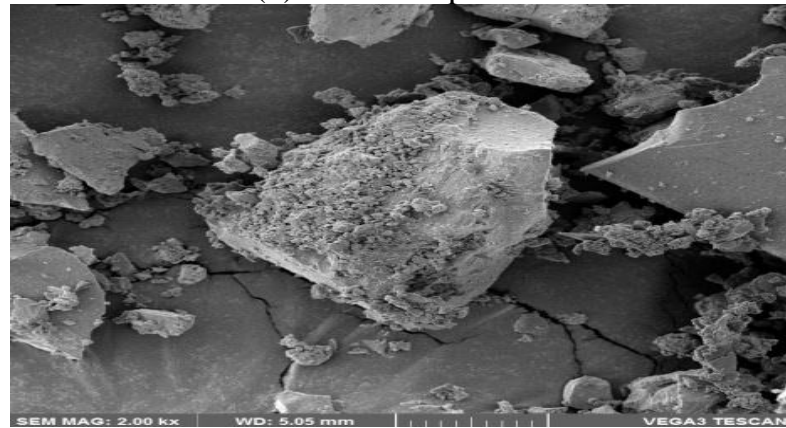

2(c) $6 \%$ Ni-doped $\mathrm{ZnS}$

Fig 2(a),(b),(c). SEM images of Nickel doped $\mathrm{ZnS}$ at the different doping concentration 
Fig.2 shows the SEM images of the Nickel doped zinc sulfide nanoparticles at various magnifications. Fig. 2(a) shows the agglomeration of the $2 \%$ nickel doped $\mathrm{ZnS}$. Fig 2(b) shows the appearance of small nano-sized grains in $4 \%$ nickel doped $\mathrm{ZnS}$, which is well agreement with the literature ${ }^{1}$ [4].Fig 2.(c) shows the cubic structure, which corresponds to the $6 \%$ dopant of nickel ion with the $\mathrm{ZnS}$. The SEM images of nickel-doped $\mathrm{ZnS}$ nanoparticles show that the particles are distributed with agglomeration with the appearance of cubical nanostructures. But as the dopant concentration increases it gives the clear images of the cubical nanostructure.

\subsection{FTIR studies}

The FTIR spectrum of the nickel-doped Zinc sulfide nanoparticles is shown in fig.3. It shows the peak at FTIR transmittance spectra of undoped and $\mathrm{Ni}^{2+}$ doped $\mathrm{ZnS}$ nanoparticles synthesized at the optimized temperature in air for $4 \mathrm{~h}$ is shown in Fig.3. The $\mathrm{ZnS}$ nanoparticles demonstrate the attributes of the formation of high virtue products and portray the peaks correspond to $\mathrm{ZnS}$. From the FTIR spectra (Fig.3), several peaks at 3400, 1566, 1341, 618 and $488 \mathrm{~cm}^{-1}$ can be observed. The broad weak peaks at $3349 \mathrm{~cm}^{-1}$ must be related to the stretching and bending modes of trace amounts of adsorbed water on the particles (Wegmuller, 1987). The peaks at 2340 and $2045 \mathrm{~cm}^{-1}$ are ascribed to the existence of $\mathrm{CO} 2$ molecules. The peak at $1384 \mathrm{~cm}^{-1}$ region could be attributed to nitrate groups (Martinez-Sabater et al., 2009). The peaks appearing at 674 and $488 \mathrm{~cm}^{-1}$ are associated with $\mathrm{Zn}-\mathrm{S}$ vibration and are characteristic of cubic ZnS (Kuppayee et al., 2011). The infrared absorption frequencies and the tentative vibrational assignments of pure and $\mathrm{Ni}$-doped $\mathrm{ZnS}$ nanoparticles are depicted in Table 2.

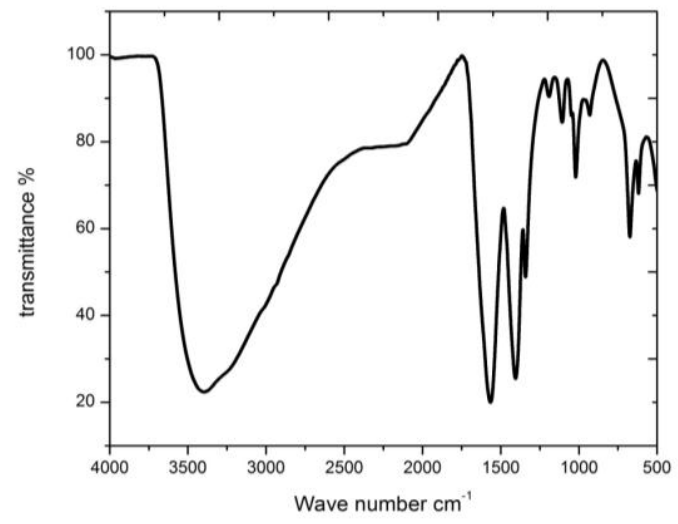

Figure 2. FTIR spectrum of Nickel doped zinc sulfide particles

Table 2. Tentative vibrational assignments of Ni-doped $\mathrm{ZnS}$ nanoparticles

\begin{tabular}{|l|l|}
\hline Wave numbers $\left(\mathrm{cm}^{-1)}\right.$ & Assignments \\
\hline 3400.63 & OH stretching \\
\hline 1566.26 & O-H Bending \\
\hline
\end{tabular}

\begin{tabular}{|l|l|}
\hline 1405.39 & $\mathrm{C}=\mathrm{O}$ bending \\
\hline 1341.46 & $\begin{array}{l}\text { Carboxyl and } \\
\text { methylene group }\end{array}$ \\
\hline 1191.52 & C-O-C \\
\hline 674.24 & Cubic ZnS \\
\hline 488.39 & $\begin{array}{l}\text { Zn-S Vibration\& } \\
\text { characteristic of } \\
\text { Cubic ZnS }\end{array}$ \\
\hline
\end{tabular}

\subsection{UV-Vis Absorption studies}

The absorption spectra are recorded in the range of 200-800 $\mathrm{nm}$. The strongest absorption peak is found at $303.5 \mathrm{~nm}$ and $301.1 \mathrm{~nm}$ for the samples of different concentrations, which is blue shifted from the absorption edge of bulk $\mathrm{ZnS}$. The blue shift due to the size reduction or due to the quantum confinement effect of the nanoparticle.[6-7].By doping with Nickel ions the absorption edge wavelength shifts towards higher wavelength.

Significant variation in absorption is observed when doping concentration is varied. The blue shift in the absorption edge is due to the quantum confinement of the excitons present in the sample, resulting in a more discrete energy spectrum of the individual nanoparticles. The effect of the quantum confinement on impurity depends upon the size of the host crystal. As the size of the host crystal decreases, the degree of confinement and its effect increases [8]. On doping $\mathrm{Ni}$ in the $\mathrm{ZnS}$ nanoparticles, the blue shift further increases, which might be due to the fact Ni forming new energy levels in the $\mathrm{ZnS}$ energy band. Using equation 2 and 3, the band-gap and average particle size of $\mathrm{ZnS}$ is found and is tabulated in Table 1.

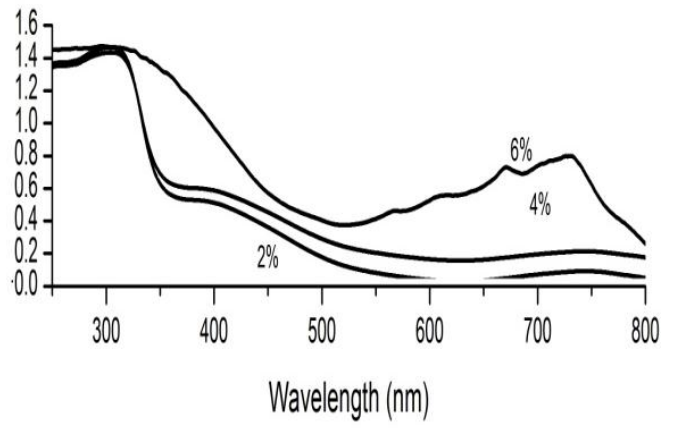

Figure 3.Absorption spectra of $\mathrm{ZnS}$ at a different dopant concentration of Nickel

The band gap energy in a nanomaterial could be obtained from the absorption maxima. According to quantum confinement theory, electrons in the conduction band and holes in the valence band are spatially confined by the potential barrier of the surface. Due to the confinement of both electrons and holes, the lowest energy optical transition from the valence to conduction band will increase in energy, effectively increasing the band gap $\mathrm{E}_{\mathrm{g} . .}$ [9] 


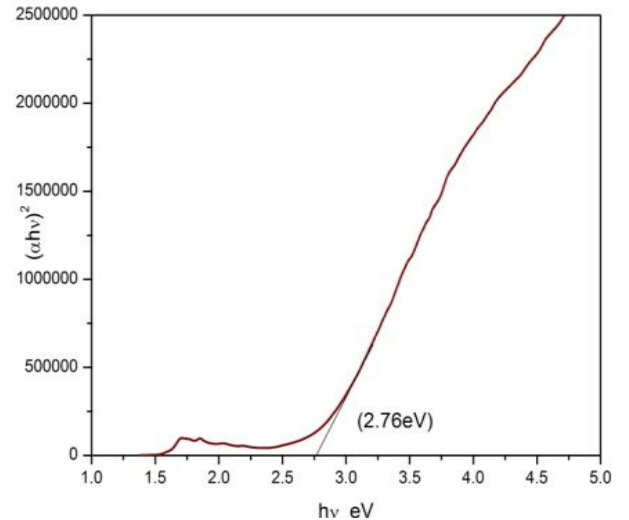

Fig 5(a). Tauc plot for $2 \%$ of Nickel doped $\mathrm{ZnS}$

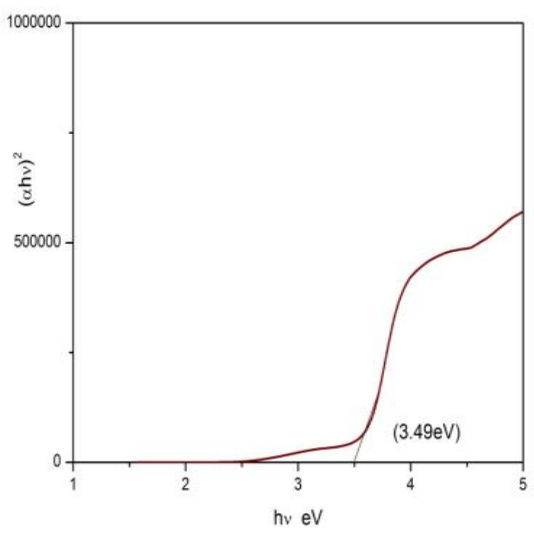

Fig 5(b).Tauc plot for $4 \%$ of Nickel doped $\mathrm{ZnS}$

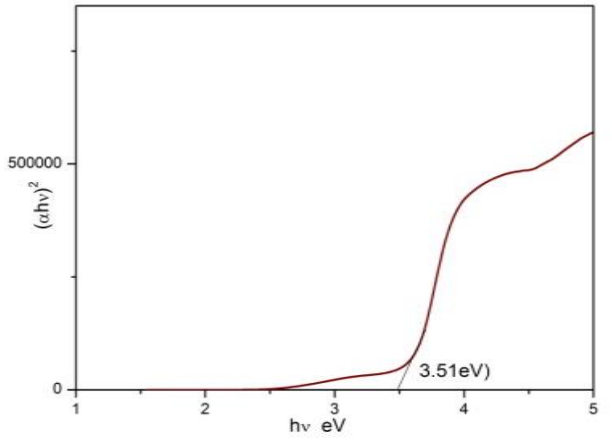

Fig 5(c).Tauc plot for $6 \%$ of Nickel doped $\mathrm{ZnS}$

The shoulder or peak of the spectra corresponds to the fundamental absorption edges in the samples, and could be used to estimate the band gap of the nanomaterial [10]. Due to quantum size effects, the band gap energy increases with decreasing particle size. [11] From the absorption peak, the optical energy band gap of $\mathrm{ZnS}$ nanostructure has been calculated using the formula

$$
\mathrm{E}_{\mathrm{ng}}=\mathrm{h} v_{\mathrm{gn}}=\mathrm{hc} / \lambda_{\mathrm{gn}}
$$

where $\mathrm{h}=$ plank's constant and $\mathrm{E}_{\mathrm{gn}}=$ energy band gap of the semiconducting nanoparticles in the optical spectra. Fig.5(a),5(b),5(c).shows the Tauc plots for 2\%, 4\%, $6 \%$ of nickel doped $\mathrm{ZnS}$ nano particles. The calculated band-gap value of the $2 \%$ nanoparticles for nickel doped $\mathrm{ZnS}$ is 2.76 $\mathrm{eV}, 4 \%$ nickel doped $\mathrm{ZnS}$ is $3.49 \mathrm{eV}$ and for $6 \%$ nickel doped $\mathrm{ZnS} 3.51 \mathrm{eV}$ which is blue shifted from that of bulk $\mathrm{ZnS}$. Increasing of band gap energies of $\mathrm{ZnS}$ nanostructures could be an indication of the quantum confinement effect due to the decreasing size of structures. The energy gap and average particle size for nickel doped $\mathrm{ZnS}$ are listed in table 1. We observed from table 1 the particle size decrease with doped $\mathrm{ZnS}$ and increasing concentration of $\mathrm{Ni}^{2+}$ ion.

3.5.Photoluminescence study

Photoluminescence (PL) of different concentration of $\mathrm{Ni}$ : $\mathrm{ZnS}$ samples are measured at room temperature using $\mathrm{F}$ 2500FL Spectrophotometer. It is observed that the PL emission band from $\mathrm{Ni}^{2+}$ doped $\mathrm{ZnS}$ sample shows emission at a visible region. (Fig. 6). Emission band with multiple peak maxima indicate the involvement of different luminescence centers in the radiative process. In the PL process, an electron from the valence band is excited across the band gap and photoexcited electron subsequently decays by a normal recombination process to some defect states.

In Ni-doped crystals, an electron may be captured by the $\mathrm{Ni}$ ${ }^{2+}$ ions in the $4 \mathrm{~T} 1$ level, from which it makes a radiative transition to the ground state 6 A 1 level. PL spectrum of $\mathrm{Ni}$ ${ }^{2+}$ doped $\mathrm{ZnS}$ nanoparticles are shown in Fig.6, It is observed that PL emission band from undoped $\mathrm{ZnS}$ nanoparticles are broadened with multiple peaks in the blue region. Peaks are found at 426,444 and $485 \mathrm{~nm}$. $\mathrm{Ni}{ }^{2+}$ doped $\mathrm{ZnS}$ nanoparticles show PL emission at 426, 448 and $486 \mathrm{~nm}$ in the visible region with an excitation wavelength of $310 \mathrm{~nm}$.

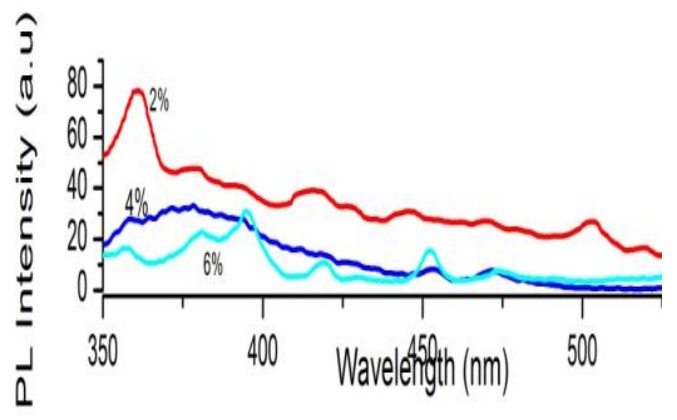
Fig 6..Photoluninance of different concentration of
Nickel doped $\mathrm{ZnS}$

3.6 Magnetic studies (VSM)

The field dependent magnetization curves for the $2 \%, 4 \%$ and $6 \%$ of $\mathrm{Ni}: \mathrm{ZnS}$ at room temperature are shown in fig.7 respectively. In II-VI nanomaterials, the nature of magnetic properties depends on the magnitude of the transition metal ion ( here nickel ion $\mathrm{Ni}^{2+}$ ) exchange coupling with the electronic levels. 


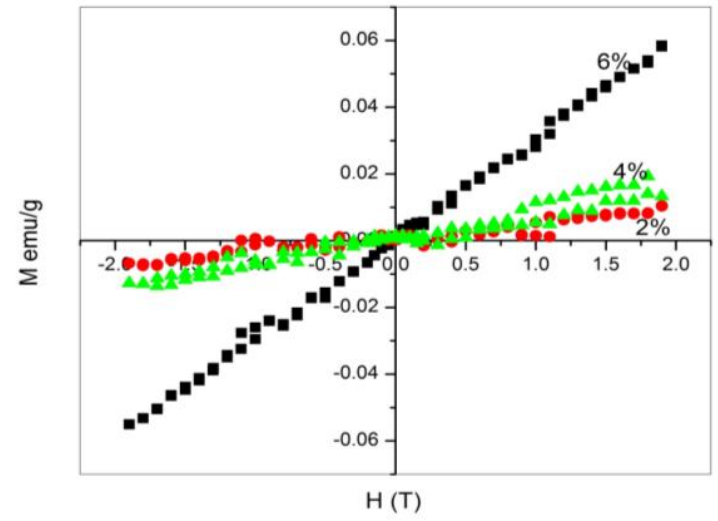

Figure 7. MH plot of $\mathrm{ZnS}$ at different concentration of Nickel

Here the ferromagnetic behavior with weak magnetic moment was observed for $2 \%$ and $4 \%$ Nickel doped $\mathrm{ZnS}$ nanoparticles. This spontaneous magnetic moment observed in $\mathrm{Ni}: \mathrm{ZnS}$ nanoparticles indicates that only a fraction of the $\mathrm{Ni}^{2+}$ ions are magnetically ordered with respect to the applied magnetic field. Here the diamagnetic nature of pure $\mathrm{ZnS}$ reduces the magnetic moment of Ni-doped $\mathrm{ZnS}$ for low concentration. A complete superparamagnetic behavior is observed for $6 \%$ nickel doped $\mathrm{ZnS}$, which reflects the contribution of a high magnetic moment for obtaining the ferromagnetic hysteresis. Here, we assume that the resulting ferromagnetic behavior is derived from the substitution of $\mathrm{Ni}^{2+}$ ions for $\mathrm{Zn}$ ions without changing its zinc blende structure.

\section{CONCLUSION}

Nickel doped $\mathrm{ZnS}$ nanoparticles were synthesized by chemical co-precipitation method with three $(2 \%, 3 \%$, and $4 \%)$ different Nickel ion $\left(\mathrm{Ni}^{2+}\right)$ concentration. XRD pattern confirms the cubic zinc blende structure of $\mathrm{ZnS}$ with a small shift in the peaks at higher angles due to the incorporation Nickel ions in the lattice. The variation in optical and luminescence properties supports the Nickel dopant incorporation in $\mathrm{ZnS}$ nanocrystals. The strong emission peak observed in the range of $303 \mathrm{~nm}$ to $308 \mathrm{~nm}$, which shows the blue shift of the particles, which may be also due to the quantum confinement effect.

The VSM study reveals the room temperature superparamagnetic nature of nickel-doped $\mathrm{ZnS}$ nanoparticles. The saturation current reaches the maximum for the $6 \%$ of nickel dopant and the for $2 \%$ and $4 \%$ the low concentrations the saturation current is very small. This means the dopant concentration increases the saturation current. This shows the presence of nickel ion may create some defects or vacancy sites which play a key role in varying the optical and magnetic properties of these nickel doped $\mathrm{ZnS}$ nanoparticles for a spintronic application.

\section{ACKNOWLEDGMENT}

The Authors are thankful to Dr. Adiraj Srinivas, Scientist E, Advanced Magnetics Group, Defence Metallurgical Research Lab (DMRL), Kanchanbagh, Hyderabad- 500058 for providing vibrating sample magnetometer study (VSM), and Dr. Sakthivel Gandhi, Department of Nanoscience and Nanotechnology, Sastra University, Tanjore, for providing SEM facilities.

\section{REFERENCES}

[1] S.A. Wolf, D.D. Awschalom, R.A. Buhrman, J.M. Daughton, S.V. Molnar, M.L. Roukes, A.Y. Chtchelkanova, D.M.Treger, "Spintronics: A Spin-Based Electronics Vision for the Future Science" 294,(2001),1488-1495.

[2] J.K. Furdyna, "diluted magnetic semiconductors" J. Appl. Phys. 64 (1988) R29-R64.

[3] Sanjeev Kumar et at,"room temperature ferro Magnetism in nickel doped Zns nano particles" Journal of Alloys and Compounds 554 (2013) 357-362.

[4] G. G. Ramteke1*, A. S. Lanje2 , D. M. Pimpalshende1,"Structural and Optical Performance of ZnS Nanoparticles Synthesized via Chemical Route" International Journal of Scientific Research in Physics and Applied Sciences Vol.6, Issue.3, pp.69- 74 June (2018).

[5] Murugadoss, G.; Rajamannan, B.; Ramaswamy, V."synthesis,characterization and optical properties of water soluble ZnS:Mn2+ nano particles" The Journal of Lumin, 2010, 130, 2032.

[6] C B Murray, D J Norris, and M G Bawendi," Synthesis and characterization of nearly monodisperse $\mathrm{CdE}(\mathrm{E}=$ sulfur, selenium, tellurium) semiconductor nanocrystallites" J. Am. Chem. Soc. 115, 8706 (1993).

[7] W Wang, I Germanenko and M Samy El-Shall, "Room-Temperature Synthesis and Characterization of Nanocrystalline CdS, ZnS, and $\mathrm{Cd}_{x} \mathrm{Zn}_{1-x} \mathrm{~S}^{\prime \prime}$ Chem. Mater. 14, 3028 (2002).

[8] S. Kumara, N. Verma, and M. Singla,"An analysis of structural and opticl properties of undoped $\mathrm{znS}$ and doped (with $\mathrm{Mn}, \mathrm{Ni}$ ) $\mathrm{ZnS}$ nano particles"Chalcogenide Letters, 8 ( 2011) 561.

[9] D. Onwudiwe and P. Ajibade," ZnS, CdS and HgS Nanoparticles via Alkyl-Phenyl Dithiocarbamate Complexes as Single Source Precursors" Int. J. Mol. Sci. , 12 (2011) 5538.

[10] T. Hoa, L. Vu, T. Canh, and N. Long,"Preparaton of ZnS nano particles by hydrothermal method", J. Phys., 187 (2009) 012081.

[11] A.Tiwari, S. Khan, and R. Kher," Surface characterization and optical properties of polyphosphate capped $\mathrm{ZnS}$ nanoparticles" Advances in Applied Science Research, 2 (2011) 105.

\section{Authors Profile}

Dr. K. Raji is a retired Associate Professor and Head of the of PG research Department of Physics, Holy Cross College,(Autonomous), Tiruchirappalli, Tamil Nadu. Shee received her Ph.D degree in 2012 from Bharadhidasan University, in metallurgy .

She is 1) Life Member of IAPT, Kanpur India 2) Life Member of PMAI, Bombay, India 3) Member in ASTM, USA 4) Executive member for the region of Tamilnadu and Pondicherry in IAPT, Kanpur and Pune. She published many papers in national and international journals.

Ms. A.Maria Bernadette Leena is persuing Ph.D under the guidance of Dr. K. Raji. Presently she is working as an Assistant professor in the PG and reseach Department of Holy Cross College,(Autonomous), Tiruchirappalli, Tamil Nadu. Her current research is focused on the nano magnetic materials and their Applications. 